\title{
AN OVERVIEW OF AUTOIMMUNITY IN IMPLANTATION FAILURE: A LITERATURE REVIEW
}

DOI: $10.36740 /$ WLek202103240

\author{
Vladyslav 0. Berestoviy', Ahmad A. Mahmood' ', Oleg 0. Berestoviy' ${ }^{2}$, Valentyna G. Ginzburg' ${ }^{1}$, Dmytro 0. Govsieiev ${ }^{3}$ \\ 'BOGOMOLETS NATIONAL MEDICAL UNIVERSITY, KYIV, UKRAINE \\ ${ }^{2}$ MATERICLINIC, KYIV, UKRAINE \\ ${ }^{3}$ KYIV STATE MATERNITY HOSPITAL №5, KYIV, UKRAINE
}

\begin{abstract}
The aim: This review was aimed to understand the role of different types of autoantibodies like antiphospholipid, antithyroid, antisperm, antinuclear, anti-ovarian autoantibodies and heat shock protein HSP 60 in the process of implantation in the normal way of conceiving and IVF and also to estimate that how the presence of these autoantibodies affect the normal pregnancy outcome.

Materials and methods: This review process performed in the obstetrics and gynaecology postgraduate department, Bogomolets national medical university, Kyiv, Ukraine. It was a review of already published papers not to need the ethical board committee's approval. By following the literature review guidelines, this paper was written and searched for relevant studies regarding autoantibodies and implantation, published in medical literature till 2020 were included in this review process. The search is done for studies published till 2020 in the English language from the Medline database, including Google Scholar, PubMed, Web of Science and Cochrane library database.

Conclusions: 0 ur recent work found that the involvement of APA, ANA and/or ATA in recipients of oocyte donations did not affect their pregnancy outcomes. Some researchers did not give any clear conclusion about these risks, and some stated that the use of some immunodepressant agents could be useful to reduce the harmful effects of these autoantibodies associated with implantation failure. Each autoantibody has a different mechanism of action to create the pathological state, some have direct effect, and some indirectly impact implantation. In future, further high-quality studies need to be performed for better understanding.
\end{abstract}

KEY WORDS: autoimmunity, immunosuppression, miscarriage, IVF failure, implantation

Wiad Lek. 2021;74(3 p.II):777-783

\section{INTRODUCTION}

The implantation process involves the interaction of the human blastocyst and the uterine epithelium [1-4]. Several autoimmune factors have been implicated in influencing implantation failure. Recent studies have investigated the contribution of autoimmune factors in implantation in women undergoing in-vitro fertilization [5-7]. Antiphospholipid antibodies more frequently identified in women planing in-vitro fertilization; their presence appears to affect the outcome of live birth rates, miscarriage, or pregnancy [8-10]. Antithyroid antibodies are commonly found in reproductive age women, but implantation rates and miscarriage rates are not connected with normal thyroid function $[11,12]$. Antinuclear antibodies may be a marker for underlying autoimmune disease when coupled with specific signs and symptoms, but low-titer antibodies do not influence in-vitro fertilization outcome [13, 14]. Fertilization failure is often associated with high titers of anti-sperm antibodies in seminal plasma, sperm, or women's mucosal immune system $[15,16]$. During implantation, growth factors may interact with adhesion molecules and other matrix-associated proteins [17]. This study mainly focuses on Implantation representing a critical developmental process characterized by the interaction of two immunologically and genetically distinct tissues.

\section{THE AIM}

This review was aimed to understand the role of different types of autoantibodies like antiphospholipid, antithyroid, antisperm, antinuclear, anti-ovarian autoantibodies and heat shock protein HSP 60 in the process of implantation in the normal way of conceiving and IVF and also to estimate that how the presence of these autoantibodies affect the normal pregnancy outcome.

\section{MATERIALS AND METHODS}

This review process performed in the obstetrics and gynaecology postgraduate department, Bogomolets national medical university, Kyiv, Ukraine. It was a review of already published papers not to need the ethical board committee's approval. By following the literature review guidelines, this paper was written and searched for relevant studies regarding autoantibodies and implantation, published in medical literature till 2020 were included in this review process. The search is done for studies published till 2020 in the English language from the Medline database, including Google Scholar, PubMed, Web of Science and Cochrane library database. The following keywords were searched: implantation, 
autoimmunity, miscarriage, infertility, spontaneous abortion, IVF, autoantibodies. The references of all main and review articles were checked to ensure the relatedness according to searched terms. The research studies that were specifically associated with our searched terms were included in this review. All collected articles were in the English language, and most females were the subjects of discussion. The studies associated with an inadequate description of the analytical method, short communications, letters and irrelevant to our searched terms were excluded. We searched the citations online, and were downloaded in one folder. The high-quality parameters were taken into account for assessing each study, i.e. study design, sample size and calculations, age of subjects, inclusion and exclusion criteria, use of control, proper utilization of statistical test and proper explaining of results. The studies which fulfil the criteria of research were selected for this review.

\section{REVIEW AND DISCUSSION}

\section{PROCESS OF IMPLANTATION}

Immunopathological mechanisms in patients with antiphospholipid syndrome resulting from binding of $\lg G$ to both platelet and endothelial membrane phospholipid may lead to uterine vessels thrombosis and vasoconstriction [18]. This relationship can cause the platelet membrane's instability and hyper aggregation by mechanisms such as protein $\mathrm{C}$ activation inhibitors for endothelial prostacyclin synthase activity [19]. Also, this may inhibit the activity of prekallikrein and activator release of endothelial plasminogen. In recent years, different types of reproductive defects have been referred to as autoimmune components. Immunological effects, including fertilization, implantation, and placental development, are significant at several stages of the reproductive cycle. Severe clinical disorder - antiphospholipid syndrome or, more simply, anti-cardiolipin syndrome - has been shown to include the development of elevated serum levels of cardiolipin autoantibodies [20].

In a relatively healthy population, $\lg G$ anti-cardiolipin autoantibodies are prevalent at 1.75 per cent. In this study, $14.3 \%$ were found to have LGG auto anti-cardiolipin compared to none in the corresponding control group. Implant failure is a chemical pregnancy in the IVF-embryo transfer (ET) system [21]. Therefore, it is tempting to conclude that, in light of the evidence previously provided here, an implantation failure may be associated with high anti-cardiolipin auto-antibodies vitamins found in the serum of these patients [22].

\section{AUTOIMMUNE FACTORS POTENTIALLY RELEVANT TO IN-VITRO FERTILIZATION FAILURE} The role of autoimmune factors in the implantation of in vitro fertilization (IVF) women has been investigated in recent studies [23].

\section{ANTIPHOSPHOLIPID ANTIBODIES}

Antiphospholipid antibodies [IgG, IgM, IgA] that respond to negatively charged phospholipids are antibodies that are acquired [24]. Antiphospholipid antibodies (APAs) associated initially with progressive slow thrombosis and placenta infarction, some of which were labelled as thrombophilic. When considering implantation and pregnancy, APAs are more properly categorized as autoimmune reality because of its complex nature [25]. HCG released from placenta inhibited in vitro migration of trophoblast, inhibited invasion and multinucleated cell formation, inhibited trophoblast cell adhesion molecules, and inhibited complement to the surface of the trophoblast, causing inflammatory reactions [26].

APAs are said to increase living birth levels combined with chronic pregnancy loss and heparin and low-dose aspirin therapy $[27,28]$. Several public reports show that the number of positive APAs is more frequent in patients who have either IVF or IVF failed instead of controls [9, 29]. Positive APAs were not associated with lower rates of pregnancy in IVF-related women. Recent findings have revealed the lack of follicular fluid APAs that do not adversely affect women undergoing IVF reproductive outcomes. Also, clinical studies of IVF-positive women with APAs show that heparin and aspirin are not improved in women with implantation or pregnancy [30].

\section{ANTITHYROID ANTIBODIES}

Severe disease, postpartum thyroiditis, and autoantibodies to thyroglobulin and thyroid peroxidase (microsomal antigen) are present in patients with Hashimoto thyroiditis. Antithyroid (ATAs) may be a marker in other autoimmune diseases or identify women who do not meet the increased demand for early pregnancy thyroid hormones [31, 32]. The pathophysiological role of ATA in pregnancy loss is based on two working hypotheses. Lifted thyroid antibodies can lead directly to pregnancy loss through biological interaction between hormones and antibodies [33]. Instead of the actual cause of pregnancy loss, ATAs may also be secondary to autoimmune diseases. Such ATAs may represent an abnormal immunological response related to pregnancy [34]. However, the future risk of miscarriage was not affected by the thyroid antibody's status in a prospective trial of 870 women with unexplained recurrent pregnancy loss with ATA and normal thyroid function [35]. Most studies indicate that $14-18 \%$ of women undergoing IVF are ATA-positive. In our extensive study, ATAs were not found more frequently in women subject to IVF (144 out of 873 or $16.4 \%$ ) than in normal controls ( 29 out of 200 or $14.5 \%$ ) [36]. No variance in results was observed in assessing the biochemical loss, clinical loss, delivery or failure rate of implantation compared to or without ATA among 873 women subject to IVF. Smaller studies have shown that women with ATA who have been IVF have experienced an increased rate of miscarriage [37]. However, the rate of delivery of levothyroxine to these women did not change. We do not test women with ATA implantation failure, nor do we think that ATA's presence in women with euthyroid changes IVF. 


\section{ANTISPERM ANTIBODIES}

Antigens that are extraneous to both male and female immune systems are present in sperm cells. In seminal plasma, male or female serum, or cervical mucus, anti-pulmonary (AP) antigens (APAs) can be induced with sperm exposure to the immune system $[16,38]$. ASAs have been identified in $10-15 \%$ of men with infertility and in $15-20 \%$ of women with unexplained infertility. The reported prevalence and suspected value depend on the population, the specimen's source (serum, cervical mucus, semen) and the test methods[39]. Antibodies are expected to interfere with the fertilization process through various mechanisms, such as sperm transport in the female genital tract, altered sperm training or acrosomal reactions, inhibiting fertilization disorders or early embryo implantation.

The sperm binding to the pellucid zones (ZP), the sperm penetration of the pellucid zone, the zoom of the reaction zone, the gamete's fusion, the embryo cleavage and the development of the embryo are possible locations where ASAs interfere with fertilization [40]. Several studies have documented the effect of sperm-bound SAs on fertilization levels after IVF [41]. Several of these studies tend to suggest a decrease in the rate of oocyte implantation or embryo fertilization with sperm-like ASAs; however, most of these studies have been retrospective in patients with inadequate single IVF fertilization $[42,43]$. In the study, pairs with low oocyte fertilization rates and ASAs were compared with average fertilisation rates without infertility [37]. Due to the sample's nature, significant differences in oocyte fertilization, implantation and pregnancy rates are expected. Checking for ASA in pairs with failed IVF fertilization may be required, but it is unnecessary to check for couples with IVF implant failure [44].

\section{ANTINUCLEAR ANTIBODIES}

Many specific antinuclear antibodies (ANAs) target molecules on the apoptotic cell surface. Both molecules have an immune function and have been postulated to lead to the abnormal release of APA or ANA in human and mouse autoimmune models, along with phospholipids in the apoptotic cell region $[45,46]$. The cause of elevated APA and ANA rates in individuals with autoimmune disorders may be a significant cell abnormality, such as increased apoptosis or decreased clearance of apoptotic cells [47]. The new analysis has shown that ANA and APA are secondary to endometriosis-induced autoimmune reactions in women with implant failure. ANAs were present in 27 of 100 endometriosis patients than 18 of 62 endometriosis-free patients [48]. Most of the studies that tested positive ANA in IVF patients suggested an increase in ANA incidence than controls; in fact, many of these results were limited to small patients and many autoimmune antibodies. The study showed improved implantation and clinical pregnancy rates of short-term immunosuppression with Prednisolone during IVF, while live birth rates differed slightly from controls [49]. A retrospective randomized clinical review of females undergoing IVF who had positive ANA shows a lack of progress in females' implantation or pregnancy with heparin and aspirin. More studies are needed to determine ANA's potential role in patients with failed implantation $[50,51]$.

\section{ANTI-OVARIAN ANTIBODIES}

Anti-ovarian antibodies (AOAs) are a heterogeneous anti-ooplasm group that includes ooplasm, pellucid region, granular membrane, theca folliculi internal cells and lutein cells [52]. Several research studies related AOA to menstrual diseases such as amenorrhea and oligomenorrhea. Some authors have suggested the prevalence of AOA in patients with failed implantation and fertilization, indicating a critical AOA role in infertility [53]. Most of the studies reported are small or non-randomized and prospective, so the role of $\mathrm{AOA}$ in reproductive failure is difficult to draw meaningful conclusions. Increased AOA prevalence in IVF failure may be associated with more widespread immune deregulation of implant failure [54]. Cross-reactions with heat-shocking protein 90-b of positive AOA sera of infertile women and others with ovarian failure. Several recent small studies have suggested that AOA may be used as a marker for assessing the use of corticosteroids in patients with previous IVF failure. Ovarian hypofunction and ovarian failure may be associated with AOA, but further studies are needed if a failed implantation is to be identified [55].

\section{ROLE OF HEAT SHOCK PROTEIN HSP 60 IN IMPLANTATION FAILURE}

Heat shock protein 60 (HSP60) is a chaperone protein involved in proteins transfer and refolding proteins. Importance of HSP60 in sperm capacitation and sperm-oocyte membrane binding facility was confirmed; therefore, in this study, the effect of HSP60 on the rate of in vitro fertilization and the cleavage rate in mouse embryo was investigated [18]. The present study demonstrated that HSP60 in low dose positively affected two-cell embryo development; however, it did not significantly affect the fertilization rate. Conversely, HSP60 had adverse effects on the fertilization and cleavage rates at higher doses $[18,56]$.

Ironically, although HSP60 did not have a low-dose effect on fertilizer, it positively affected two-cell embryos' growth. A positive effect from the two-cell stage to the blastocyst stage was observed at a low dose of cleavage but was not significant [57]. Preimplantation mouse oocyte and embryo $\mathrm{ZP}$ is permeable to macromolecules up to $170 \mathrm{kD}$, with reduced permeability at approximately $110 \mathrm{kD}$. As a result, HSP60 is easy for ZP to pass and affect the newly formed zygote with a $57-69 \mathrm{kD}$ [58]. In the early stages of embryogenesis in a natural state, HSP 60 is expressed during the final follicle maturation (Graafian follicles), indicating the positive function of HSP 60 during preimplantation [59]. The mouse embryo cleavage rate, particularly from morula to blastocyst development, was reduced when antibodies to HSP60 and HSP 70 were added to the IVF media, which strongly supports 
Table I. Implantation failure and auto-antibodies [18].

\begin{tabular}{cccc}
\hline Autoantibody & $\begin{array}{c}\text { Frequency in women undergoing in-vitro } \\
\text { fertilization compared with controls }\end{array}$ & $\begin{array}{c}\text { Association with } \\
\text { implantation failure }\end{array}$ & Other known associations \\
\hline Antiphosholipid antibodies & Increased & Unproven & Recurrent pregnancy loss \\
\hline Antithyroid antibodies & No difference & Unproven & Thyroiditis \\
\hline Antisperm antibodies & No difference & Unproven & Fertilization failure \\
\hline Antinuclear antibodies & Slightly increased & Unproven & Autoimmune disease \\
\hline Antiovarian antibodies & No difference & Unproven & Ovarian failure \\
\hline
\end{tabular}

these chaperones importance in preimplant mice [60]. Also, HSP60 is essential for its antiapoptotic and mitotic functions in early embryogenesis.

Although researchers realize, high oxygen concentrations in the IVF media may lead to apoptosis during IVF processes due to oxidation stress [61]. Therefore, it is no surprise that HSP60 has a positive effect on the growth of two cells in the embryo [62]. Therefore, unless the zygote was properly exposed to HSP60, this exogenous chaperone protein may have had a more significant impact on embryo production at the blastocyst stage [63]. Besides, HSP60 was responsive to its antiapoptotic influence, as the proportion of clogged embryos arrested decreased when $10 \mathrm{ng} / \mathrm{mL}$ of HSP60 was added to the fertilization medium [64]. HSP60 may neutralize the reactive oxygen species (ROS) in mice produced in vitro embryos, thereby preventing apoptosis stress [65].

Our primary concern for this review was to analyze the autoantibodies in relation to gynaecology and reproductive immunology. We reviewed the most common autoantibodies that affect the normal pregnancy outcome. This article highlighted several critical autoimmune factors involved in implantation that highlight their potential role in the reproduction process. Autoantibodies and early embryo mortality have a very critical role in association with implantation failure. This fact remains a critical issue facing IVF scientists struggling to implant the vast majority of embryos. The implantation failure might result from reduced uterine receptivity, embryonic defects, sperm defects, recurrence of the embryo or zones of hardening, or multifactorial causes (thin endometrium, altered expression of adhesive molecules, and possible immune factors). Our recent work found that the involvement of APA, ANA and/or ATA in recipients of oocyte donations did not affect their pregnancy outcomes (see table I).

Previously it has been suggested that APA found more frequently in individuals undergoing IVF and maybe the reason for the pregnancy loss [27, 29]. In some other studies, it was found that APA does not affect individuals undergoing IVF. Similarly, antithyroid antibodies (ATA) also responsible for miscarriage in individuals undergoing IVF. The individuals with normal thyroid function do not show any pregnancy-related complication [35]. ATA antibodies have a direct and indirect effect, so that it may be the secondary to autoimmune diseases. An antigenic substance in sperm cells triggers the immune response and production of antisperm antibodies (ASA) that cause infertility in both genders. Antinuclear antibodies (ANA) and APA play a role in implantation failure; the mechanism behind their action follows hypotheses: be they increase the process of apoptosis oy decrease the clearance of apoptotic cells. About anti-ovarian antibodies (AOA), there was a contrast in the conclusion of different researchers; some states that AOA play a role in implantation failure and leads to infertility and other mentioned that its difficult to draw some conclusion about it. Maybe this uncertainty is because of overlying multiple coexisting conditions.

The heat shock protein HSP60 in one murine research study demonstrated that it does not affect the fertilization at a low dose. In contrast, at high dose, it adversely affects the fertilization and hence implantation. Measurement of these autoantibodies clinically can be implicated as essential laboratory screening parameter before implantation in the expectant individuals to increase the ratio of positive pregnancy outcome. Increasing the awareness of this screening among the health care professional and timely diagnosis and management will develop the possibility of high implantation success ratio in expectant individual. It is not clearly understood that the presence of some autoantibodies affects normal pregnancy outcome. Researchers have different opinions about autoimmune antibodies; some suggested that they are a risk factor for implantation, and other states that their presence does not impact the normal pregnancy outcome. According to this study, the harmful effects of these autoantibodies on implantation dominate, but some uncertainties exist. In future, further studies need to be done to reveal these uncertainties and for better understanding.

\section{CONCLUSIONS}

Normal implantation of the embryo in the absence of any pathology is crucial for normal pregnancy outcome. The autoimmune antibodies are a significant risk factor for implantation failure both in normal conceiving and in IVF. The prophylactic measurement and management of these autoantibodies in the expectant individuals before conceiving may provide the opportunity to reduce the associated risks and increase the ratio of normal pregnancy outcome. Some scientists strongly suggested that the presence of autoantibodies like antiphospholipid, antithyroid,antisperm antibodies, antinuclear, anti-ovarian 
autoantibodies and heat shock protein HSP 60 are mostly associated with adverse outcome in terms of implantation, some other opposed these conclusions and stated that presence of some of these antibodies does not always lead to abnormal pregnancy outcome. Our recent work found that the involvement of APA, ANA and/or ATA in recipients of oocyte donations did not affect their pregnancy outcomes. Some researchers did not give any clear conclusion about these risks, and some stated that the use of some immunodepressant agents could be useful to reduce the harmful effects of these autoantibodies associated with implantation failure. Each autoantibody has a different mechanism of action to create the pathological state, some have direct effect, and some indirectly impact implantation. In future, further high-quality studies need to be performed for better understanding.

\section{REFERENCES}

1. Chen X., Man G.C.W., Liu Y. et al. Physiological and pathological angiogenesis in endometrium at the time of embryo implantation. Am J Reprod Immunol. 2017;78(2):e12693.

2. Sugihara K., Kabir-Salmani M., Byrne J. et al. Induction of trophinin in human endometrial surface epithelia by $\mathrm{CG} \beta$ and IL-1 $\beta$. FEBS Lett. 2008;582(2):197-202.

3. Ashary N., Tiwari A., Modi D. Embryo implantation: war in times of love. Endocrinology. 2018;159(2):1188-1198.

4. Kliman H.J., Frankfurter D. Clinical approach to recurrent implantation failure: evidence-based evaluation of the endometrium. Fertility and sterility. 2019;111(4):618-628.

5. Porcu-Buisson G., Lambert M., Lyonnet L. etal. Soluble MHCClass I chainrelated molecule serum levels are predictive markers of implantation failure and successful term pregnancies following IVF. Hum Reprod. 2007;22(8):2261-2266.

6. Di Rosa R., Ferrero S., Cifani N. et al. In vitro fertilization and autoimmunity: Evidence from an observational study. European Journal of Obstetrics \& Gynecology and Reproductive Biology. 2019;234:137142.

7. Muller V., Ob'edkova K., Krikheli I. et al. Successful pregnancy outcome in women with recurrent IVF failure and Anti-hCG autoimmunity: a report of three cases. Case reports in immunology. 2016.

8. Yoshinaga K. Review of factors essential for blastocyst implantation for their modulating effects on the maternal immune system. Semin Cell Dev Biol. 2008.

9. Hong Y.H., KimS.J., Moon K.Y.etal. Impact of presence of antiphospholipid antibodies on in vitro fertilization outcome. Obstetrics \& gynecology science. 2018;61(3):359.

10. El Hasbani G., Khamashta M., Uthman I. Antiphospholipid syndrome and infertility. Lupus. 2020;29(2):105-117.

11. Chaouat G., Ledée-Bataille N., Dubanchet $S$. Immune cells in uteroplacental tissues throughout pregnancy: a brief review. Reproductive biomedicine online. 2007;14(2):256-266.

12. Leiva P., Schwarze J.E., Vasquez P. et al. There is no association between the presence of antithyroid antibodies and increased reproductive loss in pregnant women after ART: a systematic review and meta-analysis. JBRA assisted reproduction. 2017;21(4):361.

13. Cavalcante M.B., Cavalcante C.T.M.B., Sarno M. et al. Antinuclear antibodies and recurrent miscarriage: Systematic review and metaanalysis. Am J Reprod Immunol. 2020;83(3):e13215.
14. Fan J., Zhong Y., Chen C. Impacts of anti-dsDNA antibody on in vitro fertilization-embryo transfer and frozen-thawed embryo transfer. Journal of immunology research. 2017.

15. Shibahara H., Wakimoto Y., Fukui A. et al. Anti-sperm antibodies and reproductive failures. Am J Reprod Immunol. 2020:e13337.

16. As V., Dhama K., Chakraborty S. et al. Role of antisperm antibodies in infertility, pregnancy, and potential for contraceptive and antifertility vaccine designs: research progress and pioneering vision. Vaccines. 2019;7(3):116.

17. KuttehW.H. Antiphospholipid antibody-associated recurrent pregnancy loss: treatment with heparin and low-dose aspirin is superior to lowdose aspirin alone. American journal of obstetrics and gynecology. 1996;174(5):1584-1589.

18. Abdi Z., Mohsenzadeh S., Anarkooli I.J. et al. The effect of hsp60 on fertilization and pre-implantation embryo development in mice: an in vitro study. Acta Endocrinologica (Bucharest). 2019;15(2):153.

19. ClineA.M., KuttehW.H. Is there a role of autoimmunity in implantationfailure after in-vitro fertilization? Curr Opin Obstet Gynecol. 2009;21(3):291-295.

20. Zhong Y-P., Ying Y., Wu H-T. et al. Relationship between antithyroid antibody and pregnancy outcome following in vitro fertilization and embryo transfer. Int J Med Sci. 2012;9(2):121.

21. Carp H.J., Selmi C., Shoenfeld Y. The autoimmune bases of infertility and pregnancy loss. J Autoimmun. 2012;38(2-3):J266-J274.

22. Ebrahimi M., Asbagh F.A. The role of autoimmunity in premature ovarian failure. Iranian journal of reproductive medicine. 2015;13(8):461.

23. Birkenfeld A., Mukaida T., Minichiello L. et al. Incidence of autoimmune antibodies in failed embryo transfer cycles. Am J Reprod Immunol. 1994;31(2-3):65-68.

24. Tartakovsky B., Bermas B.L., Sthoeger Z. et al. Immunology: Defective maternal-fetal interaction in a murine autoimmune model. Hum Reprod. 1996;11(11):2408-2411.

25. Stern C., Chamley L., Hale L. et al. Antibodies to $\beta 2$ glycoprotein I are associated with in vitro fertilization implantation failure as well as recurrent miscarriage: results of a prevalence study. Fertility and sterility. 1998;70(5):938-944.

26. Geva E., Amit A., Lerner-Geva L. et al. Autoimmunity and reproduction. Fertility and Sterility. 1997;67(4):599-611.

27. KholeV. Does ovarian autoimmunity play a role in the pathophysiology of premature ovarian insufficiency? Journal of mid-life health. 2010;1(1):9.

28. Saccone G., Berghella V., Maruotti G.M. et al. Antiphospholipid antibody profile based obstetric outcomes of primary antiphospholipid syndrome: the PREGNANTS study. American journal of obstetrics and gynecology. 2017;216(5):525.

29. Kokcu A., Yavuz E., Celik H. et al. A panoramic view to relationships between reproductive failure and immunological factors. Arch Gynecol Obstet. 2012;286(5):1283-1289.

30. Kwak-Kim J., Skariah A., Wu L. et al. Humoral and cellular autoimmunity in women with recurrent pregnancy losses and repeated implantationfailures: A possible role of vitamin D. Autoimmunity reviews. 2016;15(10):943-947.

31. Kikuchi K., Shibahara H., Hirano Y. et al. Antinuclear antibody reduces the pregnancy rate in the first IVF-ET treatment cycle but not the cumulative pregnancy rate without specific medication. Am J Reprod Immunol. 2003;50(4):363-367.

32. Li C., Zhou J., Huang Z. et al. The Clinical Value and Variation of Antithyroid Antibodies during Pregnancy. Dis Markers. 2020.

33. Xie J., Jiang L., Sadhukhan A. et al. Effect of antithyroid antibodies on women with recurrent miscarriage: A meta-analysis. Am J Reprod Immunol. 2020;83(6):e13238. 
34. Monteleone P., Parrini D., Faviana P. et al. Female infertility related to thyroid autoimmunity: the ovarian follicle hypothesis. Am J Reprod Immunol. 2011;66(2):108-114.

35. Haller-Kikkatalo K., Altmaee S., Tagoma A. et al. Autoimmune activation toward embryo implantation is rare in immune-privileged human endometrium. Semin Reprod Med. 2014.

36. Kling C., Steinmann J., Westphal E. et al. Adverse effects of intradermal allogeneic lymphocyte immunotherapy: acute reactions and role of autoimmunity. Hum Reprod. 2006;21(2): 429-435.

37. Tomassetti C., Meuleman C., Pexsters A. et al. Endometriosis, recurrent miscarriage and implantation failure: is there an immunological link? Reproductive biomedicine online. 2006;13(1):58-64.

38. Gleicher N., Weghofer A., Barad D.H. Cutting edge assessment of the impact of autoimmunity on female reproductive success. J Autoimmun. 2012;38(2-3):J74-J80.

39. Matteo M., Greco P., Setti P.L. et al. Preliminary evidence for high antiPLAC1 antibody levels in infertile patients with repeated unexplained implantation failure. Placenta. 2013;34(4):335-339.

40. Fawzy M., El-Refaeey A-A.A. Does combined prednisolone and low molecular weight heparin have a role in unexplained implantation failure? Arch Gynecol Obstet. 2014;289(3):677-680.

41. Margalioth E., Ben-Chetrit A., Gal M. et al. Investigation and treatment of repeated implantation failure following IVF-ET. Hum Reprod. 2006;21(12):3036-3043.

42. Fatemi H., Popovic-Todorovic B. Implantation in assisted reproduction: a look at endometrial receptivity. Reproductive biomedicine online. 2013;27(5):530-538.

43. Ya Sin A.L., Ya Sin A.L., Ba Sha W.S. The epidemiology of anti-sperm antibodies among couples with unexplained infertility in North West Bank, Palestine. Journal of clinical and diagnostic research: JCDR. 2016;10(3):QC01.

44. Bashiri A., Halper K.I., Orvieto R. Recurrent Implantation Failure-update overview on etiology, diagnosis, treatment and future directions. Reprod Biol Endocrinol. 2018;16(1):121.

45. Lee Y.L., Ng H.P., Lau K.S. et al. Increased fetal abortion rate in autoimmune thyroid disease is related to circulating TPO autoantibodies in an autoimmune thyroiditis animal model. Fertility and Sterility. 2009;91(5):2104-2109.

46. Deroux A., Dumestre-Perard C., Dunand-Faure C. et al. Female infertility and serum auto-antibodies: a systematic review. Clin Rev Allergy Immunol. 2017;53(1):78-86.

47. Raziel A., Schachter M., Strassburger D. et al. Favorable influence of local injury to the endometrium in intracytoplasmic sperm injection patients with high-order implantation failure. Fertility and sterility. 2007;87(1):198-201.

48. Roca V.I., Calafat M.J., Larocca L. et al. Potential immunomodulatory role of VIP in the implantation sites of prediabetic nonobese diabetic mice. 2009.

49. Ghazeeri G.S., Kutteh W.H. Autoimmune factors in reproductive failure. Curr Opin Obstet Gynecol. 2001;13(3):287-291. doi: 10.1097/00001703200106000-00007.

50. Li Y., Wang Y., Lan Y. et al. Antinuclear antibodies in follicular fluid may reduce efficacy of in vitro fertilization and embryo transfer by invading endometrium and granular cells. Am J Reprod Immunol. 2020;84(4):e13289.

51. Ola B., Li T-C. Implantation failure following in-vitro fertilization. Curr Opin Obstet Gynecol. 2006;18(4):440-445.
52. Bizjak M., SelmiC., Praprotnik S. et al. Silicone implants and lymphoma: the role of inflammation. J Autoimmun. 2015;65:64-73.

53. NG S.C., Gilman-Sachs A., Thaker P. et al. Expression of intracellular Th1 and Th2 cytokines in women with recurrent spontaneous abortion, implantation failures after IVF/ET or normal pregnancy. Am J Reprod Immunol. 2002;48(2):77-86.

54. Cohen Tervaert J.W., Colaris M.J., van der Hulst R.R. Silicone breast implants and autoimmune rheumatic diseases: myth or reality. Curr Opin Rheumatol. 2017;29(4):348-354.

55. Levi Setti P., Colombo G., Savasi V. et al. Implantation failure in assisted reproduction technology and a critical approach to treatment. Ann NY Acad Sci. 2004;1034(1):184-199.

56. Mekinian A., Cohen J., Alijotas-Reig J. et al. Unexplained recurrent miscarriage and recurrent implantation failure: is there a place for immunomodulation? Am J Reprod Immunol. 2016;76(1):8-28.

57. Ly D.K., Aziz N., Safi J. et al. Evidence-based management of infertile couples with repeated implantation failure following IVF. Current Women's Health Reviews. 2010;6(3):200-218.

58. Iborra A., Palacio J.R., Martínez P. Oxidative stress and autoimmune response in the infertile woman. Immunology of Gametes and Embryo Implantation. 88: Karger Publishers; 2005; p. 150-162.

59. Geva E., Amit A., Lerner-Geva L. et al. Autoimmune disorders: another possible cause for in-vitro fertilization and embryo transfer failure. Hum Reprod. 1995;10(10):2560-2563.

60. Sthoeger Z.M., Mozes E., Tartakovsky B. Anti-cardiolipin antibodies induce pregnancy failure by impairing embryonic implantation. Proceedings of the National Academy of Sciences. 1993;90(14):64646467.

61. Gajbhiye R., Suryawanshi A., Khan S. et al. Multiple endometrial antigens are targeted in autoimmune endometriosis. Reproductive BioMedicine Online. 2008;16(6):817-824.

62. Potdar N., Gelbaya T.A., Konje J.C. et al. Adjunct low-molecular-weight heparin to improve live birth rate after recurrent implantation failure: a systematic review and meta-analysis. Human reproduction update. 2013;19(6):674-684.

63. KushnirV.A., Solouki S., Sarig-Meth T. et al. Systemic inflammation and autoimmunity in women with chronic endometritis. American Journal of Reproductive Immunology. 2016;75(6):672-677.

64. Özkurt Z., Kazazoğlu E. Zirconia dental implants: a literature review. J Oral Implantol. 2011;37(3):367-376. doi: 10.1563/aaid-joi-d-09-00079.

65. Van Mourik M.S., Macklon N.S., Heijnen C.J. Embryonic implantation: cytokines, adhesion molecules, and immune cells in establishing an implantation environment. Journal of leukocyte biology. 2009;85(1):419.

The article was performed in framework of research "Perinatal-Maternal Aspects in Obstetric Practice and Current Issues in Women's Health Immunology", (2019-2021, № state registration 0119U100601). We will like to acknowledge Bogomolets National Medical University's librarian team for helping us in this review process.

\section{ORCID and contributionship:}

Vladyslav O. Berestoviy-0000-0002-5880-770X ${ }^{A, C, D}$

Ahmad A. Mahmood-0000-0002-6642-2324 ${ }^{A, B, D}$

Oleg O. Berestoviy - 0000-0002-5118-5530 A,C,E

Valentyna G. Ginzburg-0000-0001-6667-1913 ${ }^{B, C, F}$

Dmytro O. Govsieiev-0000-0001-9669-0218 C,E,F 


\section{Conflict of interest:}

The Authors declare no conflict of interest.

\section{CORRESPONDING AUTHOR}

\section{Vladyslav 0. Berestoviy}

Bogomolets National Medical University, Kyiv, Ukraine

2 Lobanovskoho Avenue, 03037 Kyiv, Ukraine

tel: +380934707674

e-mail:vladberestovoy@gmail.com

Received: 28.11 .2020

Accepted: 10.03.2021

A - Work concept and design, B - Data collection and analysis, C - Responsibility for statistical analysis,

D-Writing the article, $\mathbf{E}$-Critical review, $\mathbf{F}$ - Final approval of the article 